\title{
Responses of Coronary Arteries of Cardiac Transplant Patients to Acetylcholine
}

\author{
R. David Fish, Elizabeth G. Nabel, Andrew P. Selwyn, Paul L. Ludmer, Gilbert H. Mudge, James M. Kirshenbaum, \\ Frederick J. Schoen, R. Wayne Alexander, and Peter Ganz \\ Cardiovascular Division, Departments of Medicine and Pathology, Brigham and Women's Hospital and Harvard Medical School, \\ Boston, Massachusetts 02115
}

\begin{abstract}
Accelerated coronary atherosclerosis is a major cause of graft failure after heart transplantation. Graft atherosclerosis is typically diffuse and difficult to detect even with coronary arteriography. Recently, acetylcholine was shown to dilate blood vessels by releasing a vasorelaxant substance from the endothelium (endothelium-derived relaxing factor). We have demonstrated paradoxical vasoconstriction induced by acetylcholine both early and late in the course of coronary atherosclerosis in patients, suggesting an association of endothelial dysfunction and atherosclerosis. In this report, we tested the hypothesis that coronary arteries of heart transplant patients can show endothelial dysfunction before or in the early stages of angiographically evident coronary atherosclerosis. Acetylcholine was infused into the left anterior descending artery of 13 heart transplant patients at $12(n=9)$ and $24(n=4)$ mo after transplantation. Vascular responses were evaluated by quantitative angiography. Among patients with angiographically smooth coronary arteries, relatively few (6/25) arterial segments had preserved vasodilator responses, while the majority failed to dilate $(10 / 25)$ or paradoxically constricted (9/25). Angiographically irregular coronary arteries were present in three patients, in whom $8 / 10$ segments showed marked paradoxical constriction and the remaining $2 / 10$ failed to dilate. Only 1 of 13 patients retained appropriate dilation to acetylcholine in all segments. Nitroglycerin, which acts directly on vascular smooth muscle, dilated nearly all segments. No clinical features of the patients, including myocardial rejection appeared to correlate with the impaired functional response of vessels. Thus impaired response to acetylcholine is a common early finding in heart transplant patients and emphasizes the potential importance of endothelial dysfunction in the development of atherosclerosis.
\end{abstract}

\section{Introduction}

Accelerated coronary atherosclerosis is a major cause of graft failure and death in cardiac transplant patients (1-3). Improvements in immunosupression regimens and clinical management have enhanced long-term survival, but graft atherosclerosis occurs in 40-50\% of patients surviving $5 \mathrm{yr}(1-3)$. This disease leads to the familiar clinical sequelae of myocardial infarction, ventricular arrythmias, congestive heart failure, and sudden death, but not angina for this patient popula-

\footnotetext{
Address reprint requests to Dr. Ganz.

Received for publication 19 May 1987 and in revised form $25 \mathrm{Au}$ gust 1987.
}

\section{J. Clin. Invest.}

(C) The American Society for Clinical Investigation, Inc.

$0021-9738 / 88 / 01 / 0021 / 11 \$ 2.00$

Volume 81, January 1988, 21-31 tion has denervated hearts. Thus, clinical detection of ischemia may be delayed until an advanced stage of the disease has developed. Routine annual coronary angiography typically has been employed to monitor disease in epicardial coronary arteries, but a number of reports point to the difficulty in detecting graft atherosclerosis due to the diffuse and concentric nature of the disease process and the consequent absence of adjacent normal segments to provide a standard by which to judge stenosis.

Recently, Furchgott $(4,5)$ and others $(6-8)$ have shown that vasodilation in response to a number of agents, including the muscarinic cholinergic agonist, acetylcholine, is caused indirectly by stimulating the release of an endothelium-derived relaxing factor(s). Studies from our laboratory (9) and by others (10) have suggested that the functional response to the endothelium-dependent dilator acetylcholine is abnormal in the presence of coronary atherosclerosis and that paradoxical vasoconstriction is observed in both early and advanced disease. The purpose of this study was to evaluate the hypothesis that the coronary arteries of heart transplant patients may be functionally disturbed, even when angiographically normal or near normal. This hypothesis was tested by examining angiographically the responses of coronary arteries of cardiac homografts to the intracoronary administration of the endothelium-dependent dilator acetylcholine and the direct smooth muscle dilator nitroglycerin. The vasomotor response and angiographic appearance of the arteries were also considered in relationship to the clinical profile of each patient to gain insights into risk factors that may contribute to the development of vascular disease.

\section{Methods}

Patient selection. 13 consecutive patients who had undergone cardiac transplantation $1 \mathrm{yr}(n=9)$ and $2 \mathrm{yr}(n=4)$ previously were studied during routine annual catheterization that included coronary arteriog. raphy, left ventriculography, and right ventricular biopsy. The studies were approved by the Committee for the Protection of Human Subjects from Research Risk.

Study design. The study protocol was carried out as previously described (9). Briefly, after the completion of diagnostic catheterization, an additional 5,000 $\mathrm{U}$ of heparin were administered, and a number 8 French L 4 Judkins-type guiding catheter was introduced into the left main coronary artery. A number 2 French coronary infusion catheter (American Edwards, Santa Ana, CA; or Advanced Catheter Systems, Mountain View, CA) was advanced through the guiding catheter into the proximal portion of the left anterior descending for administration of vasoactive agents. This artery was chosen to avoid the known propensity of acetylcholine to cause bradyarrythmias due to its effects on the cardiac conduction system, the blood supply of which derives largely from the right and left circumflex coronary arteries. Prophylactically, a number 5 French bipolar pacing catheter was placed in the right ventricular apex and set in a demand mode to prevent the heart rate from slowing to below 40 beats $/ \mathrm{min}$.

Serial 2-min intracoronary infusions of vasoactive agents were administered in the following sequence: control 1 ( $5 \%$ dextrose in water); 
vehicle control 2 ( $5 \%$ dextrose in water plus 0.005 percent mannitol); graded concentrations of acetylcholine (to achieve estimated final blood concentrations in the left anterior descending coronary artery of $10^{-9}, 10^{-8}, 10^{-7}$, and $\left.10^{-6} \mathrm{M}\right)$, and repeat control $3(5 \%$ dextrose in water). The final coronary blood concentrations of acetylcholine were estimated with the assumption that blood flow in the left anterior descending artery was $80 \mathrm{ml} / \mathrm{min}$ (11).

Acetylcholine infusions were terminated either when the largest dose $\left(10^{-6} \mathrm{M}\right)$ was reached or when vessel occlusion occurred. Lastly, $40 \mu \mathrm{g}$ of nitroglycerin were administered over a period of $2.5 \mathrm{~min}$ into with a Harvard pump to keep the infusion rates under $1 \%$ of the estimated coronary blood flow. At the end of each infusion, coronary arteriography was performed in biplane orthogonal views with the use of a power injection of nonionic contrast medium (Iohexol Omnipaque; Winthrop-Bream, New York). Throughout each infusion, the heart rate, systemic arterial pressure, and electrocardigram (leads 1 , aVF, and V2) were monitored continuously. No complications were encountered as a result of these infusions.

Quantitative coronary angiography. Quantitative coronary angiography was performed by a previously validated technique (12). Nonionic contrast medium was injected into the left coronary artery at a rate of 5-6 ml/s to a total of 8-9 ml with the use of a power injector (Medrad, Pittsburgh, PA) to optimize the quality and reproducibility of the injections (13). A biplane system, (Polydiagnost-C; Philips Medical Systems, Inc., Shelton, CT) was set up to allow two image intensifiers to be positioned so that the center of each field of view was in line with a single position in space (isocenter).

Technically suitable single-plane coronary angiograms were selected from the biplane views for analysis. Where available, proximal, midvessel, and distal segments of the left anterior descending coronary artery that were free of side branches and distal to the infusion catheter were selected for quantitative analysis. Where analysis revealed functionally distinct segment subregions, the segment was subdivided. The epicardial coronary artery segment under study was centered, and the single-frame cine image was digitized with the use of a MicroVAX II computer (Digital Equipment Corp., Maynard, MA) connected to a video camera (Cohu Inc., San Diego, CA) and a (Recognition Concepts, Inc., Incline Village, NV) video interface. Four to five cine frames in end-diastole were scanned and averaged, with two anatomi$\mathrm{cal}$ references to ensure reproducible positioning of the frames. Twoline profile averaging was used to minimize anatomical noise and four video scans were summed to reduce video noise. Calibrated grids in the field of view were used to scale the data from pixels to millimeters. A series of measurements of diameter were recorded for each pixel line for the length of the arterial segment, and the data were also displayed in the form of a graph showing diameter versus length. Fixed coordinates were used to reproduce these regions of interest in repeated measurements to assess serial changes.

Statistical analysis. The digitized cine frames for each infusion were summed and averaged along the segment profile to give a mean diameter and standard deviation at each point. A single mean and standard deviation for the segment (at each infusion) was obtained by averaging each of these measures along the segment profile. Where an responses as compared to control, pairwise $t$ tests were applied to compare each graded infusion to control according to the Bonferroni procedure so that the summed errors of measurement did not exceed 0.05 , allowing rejection of the null hypothesis at the 0.95 confidence level for significant responses. Maximum segment responses were grouped by these tests into dilators $(P<0.05)$, constrictors $(P<0.05)$, as percent change of mean maximal response as compared to control, and a mean and standard deviation for each group is shown. Nitroglycerin (NTG) responses, as compared to the preceding repeat control 3 cance was assumed when the null hypothesis could be rejected at the 0.95 confidence level. the left anterior descending coronary artery. Infusions were carried out analysis of variance showed significant variation among Ach infusion and unchanged segments $(P>0.05)$. The graphed data were expressed responses, were assessed using the pooled $t$ test, and statistical signifi-

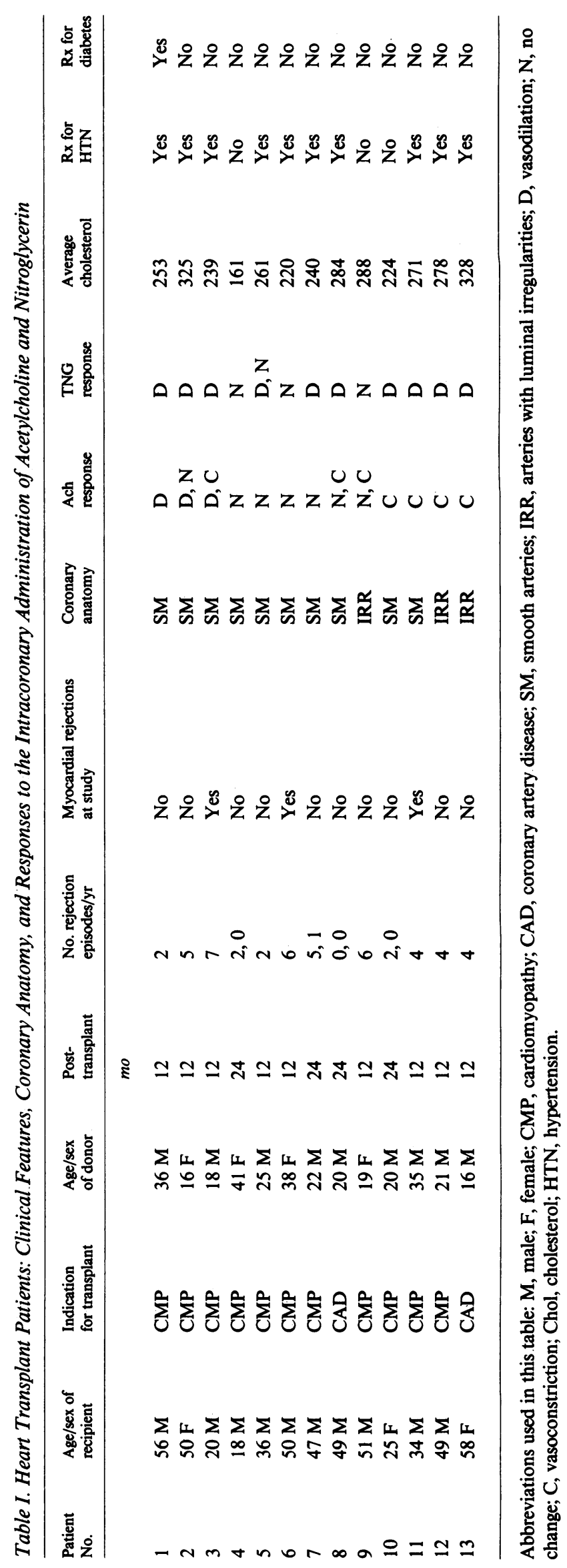



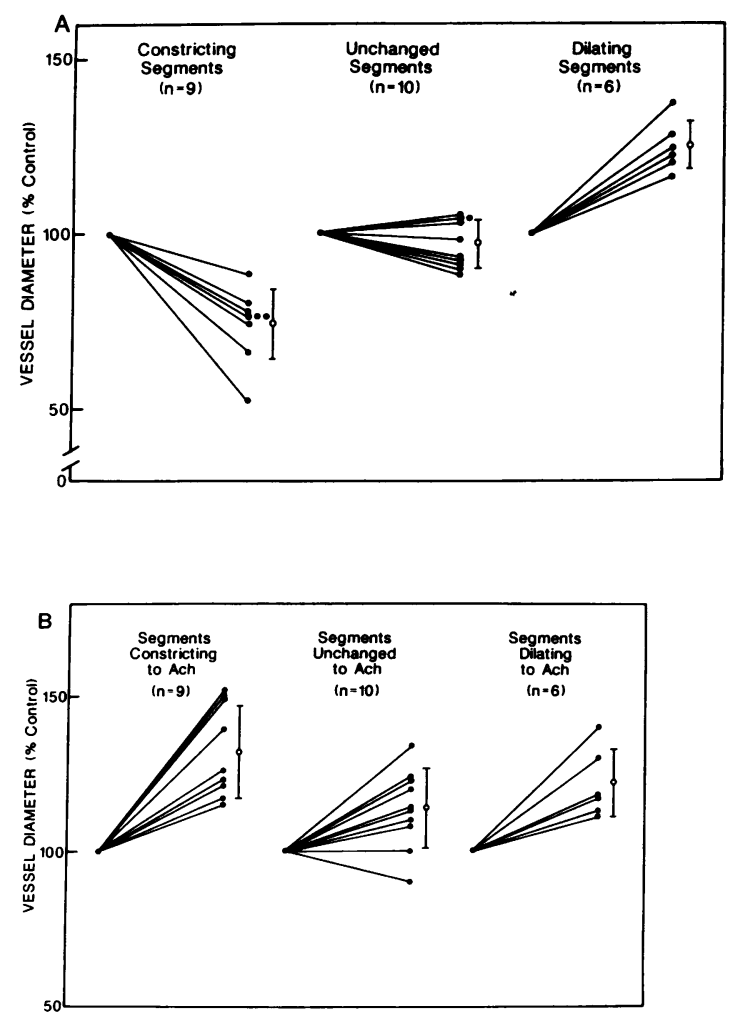

Figure 1. (A) Responses to acetylcholine of angiographically smooth coronary arteries. $(B)$ Responses to nitroglycerin of angiographically smooth coronary arteries. Mean \pm SD is shown for each group.

Clinical correlations. Clinical characteristics were examined for each patient and correlated with vasomotor responses (Table I). Features studied included the number of previous episodes of myocardial rejection of sufficient severity to require an increase in immunosuppressive therapy. In general, myocardial biopsies during such episodes revealed an interstitial mononuclear (predominantly lymphocytic) inflammatory response with evidence of myocyte necrosis.

\section{Results}

Systemic hemodynamics. Infusion of acetylcholine up to a final estimated concentration of $10^{-6} \mathrm{M}$ or infusion of $40 \mu \mathrm{g}$ of nitroglycerin directly into the left anterior descending coronary artery had no effect on the heart rate or systemic arterial pressure in any of the 13 patients.

The mean $( \pm \mathrm{SD})$ heart rates and systolic arterial pressures were $87 \pm 8$ beats/min and $131 \pm 22 \mathrm{mmHg}$ during each of the three control infusions, $86 \pm 9$ beats/min and $131 \pm 22 \mathrm{mmHg}$ with the highest dose of acetylcholine, and $86 \pm 10$ beats $/ \mathrm{min}$ and $124 \pm 23 \mathrm{mmHg}$ after the administration of nitroglycerin.

Responses of coronary arteries. Two groups of patients were identified by a consensus of three experienced angiographers who were not familiar with the acetylcholine and nitroglycerin responses. 10 patients were judged to have entirely smooth coronary arteries without any intimal irregularities. Three patients had intimal irregularities $(<50 \%$ luminal diameter narrowing) in the left anterior descending artery. One of these three patients also had a $80 \%$ luminal diameter narrowing in the circumflex artery.
25 segments of the left anterior descending artery were analyzed in 10 patients with angiographically smooth coronary arteries. Fig. $1 A$ shows the maximum response of each segment to the administered acetylcholine. The responses of the smooth vessels were heterogeneous with nine segments paradoxically constricting by $26 \pm 10 \%(P<0.05), 10$ segments demonstrating no change (see definition in Methods) and only 6 segments expressing normal physiologic dilation $(25 \pm 7 \%)(P$ $<0.05$ ). The responses of these 25 segments to nitroglycerin are shown in Fig. $1 B$. Nearly all $(22 / 25)$ segments dilated, and in particular, all segments constricting to acetylcholine dilated in response to nitroglycerin. Three segments in three patients that did not dilate in response to nitroglycerin also did not respond to acetylcholine.

7 of 10 patients had similar responses to acetylcholine in all segments while 3 patients demonstrated heterogeneous responses, 1 with both constricting and unchanged segments, 1 with dilating and unchanged segments, and 1 with dilating and
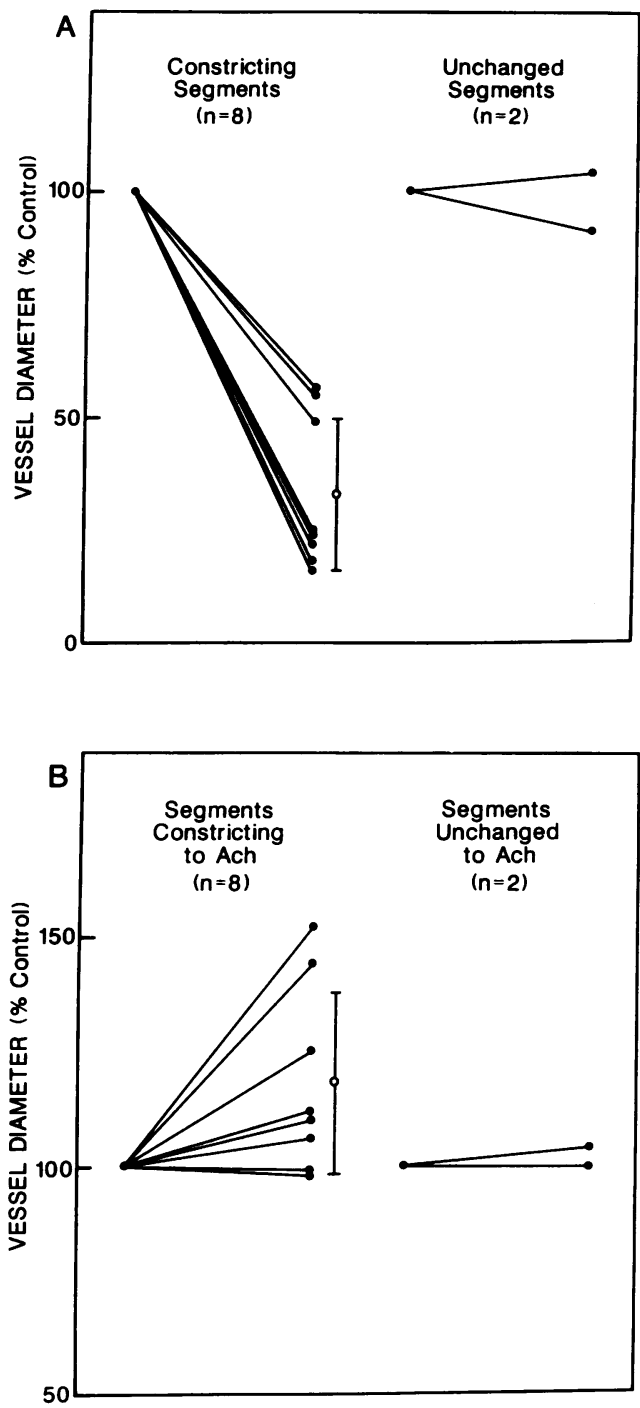

Figure 2. (A) Responses to acetylcholine of coronary arteries with angiographic luminal irregularities. $(B)$ Responses to nitroglycerin of coronary arteries with angiographic luminal irregularities. Mean \pm SD is shown for each group. 

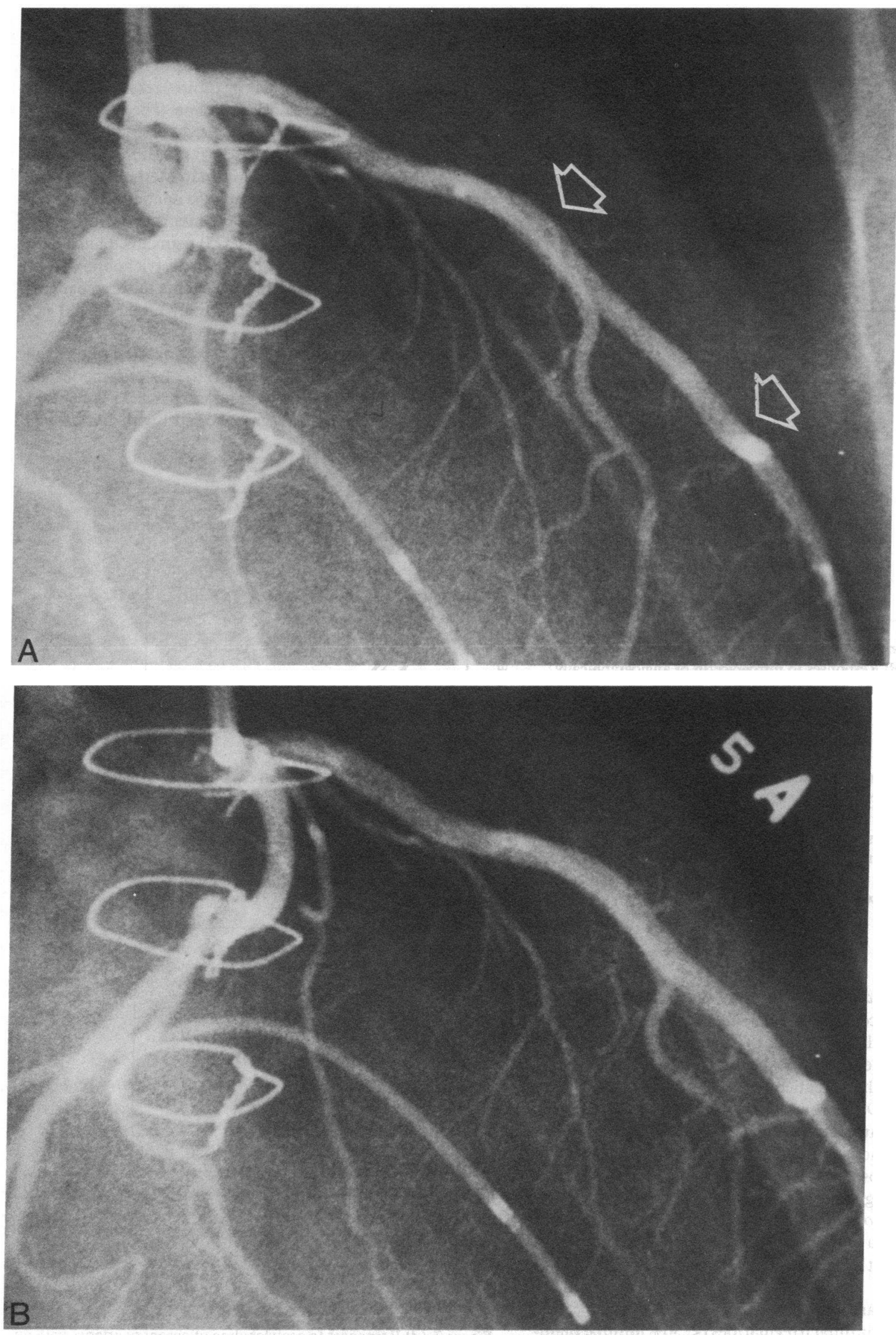

Figure 3. Coronary arteriograms in a patient with angiographically smooth coronary arteries and a dilator response to acetylcholine. $(A)$ Left anterior descending coronary artery (arrows) under baseline conditions. (B) Dilation in response to $10^{-6} \mathrm{M}$ acetylcholine. $(C)$ Dilation in response to $40 \mu \mathrm{g}$ of nitroglycerin. 


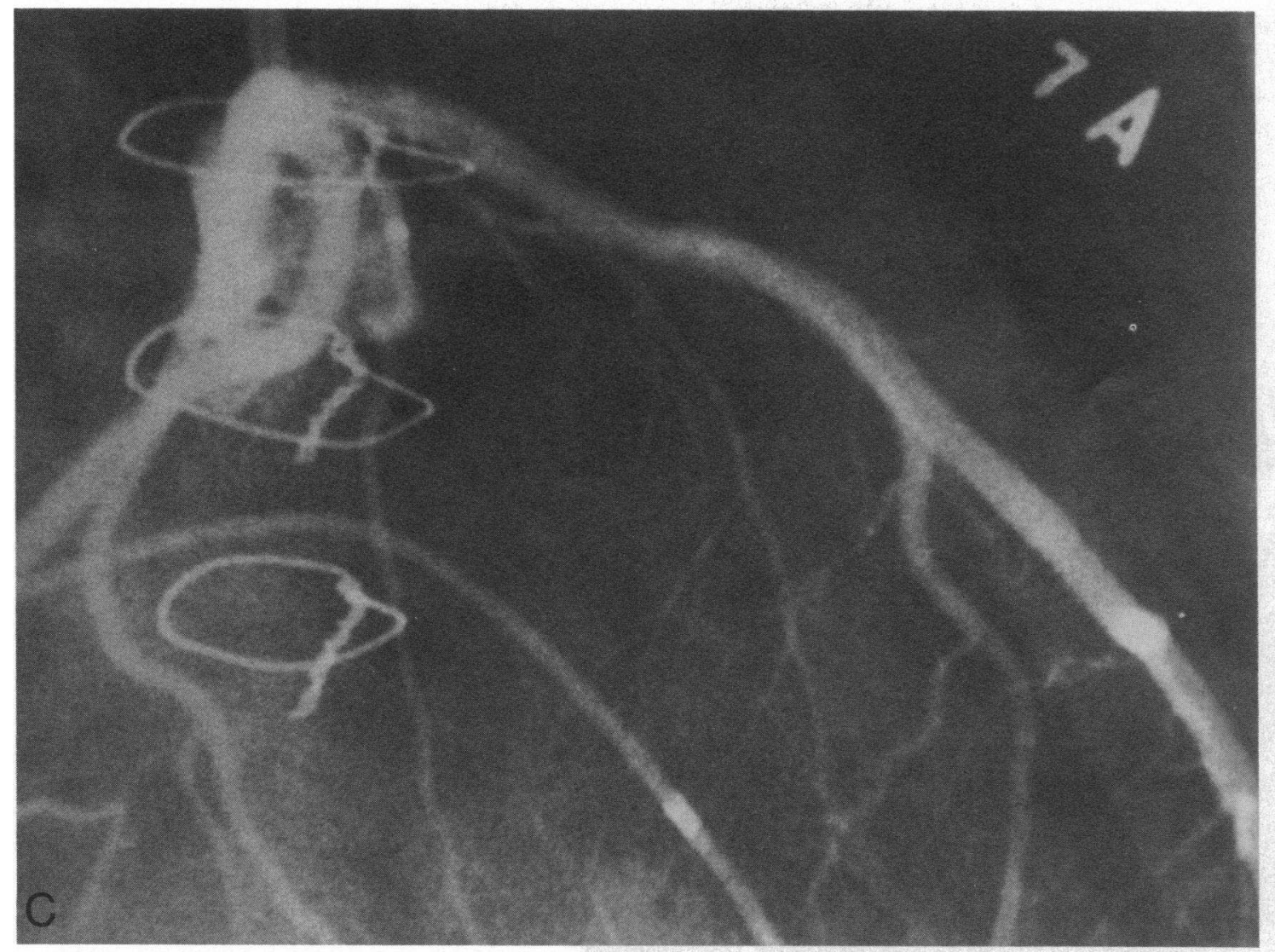

Figure 3 (Continued)

constricting segments. Only 1 of 10 patients with smooth vessels retained appropriate dilation to acetylcholine in all segments. Coronary arteriograms of a patient demonstrating dilator responses to both acetylcholine and nitroglycerin are shown in Fig. 3, while coronary arteriograms of a patient with smooth vessels demonstrating a paradoxical constriction to acetylcholine and preserved dilation to nitroglycerin are shown in Fig. 4.

10 segments were analyzed in three patients with angiographically irregular left anterior descending coronary arteries (Fig. $2 \mathrm{~A}$ ). Eight segments markedly constricted in response to acetylcholine $(67 \pm 17 \%$ reduction in luminal diameter) $(P$ $<0.05)$. In two patients acetylcholine caused virtual occlusion of the vessel at $10^{-7} \mathrm{M}$ before reaching the maximal dose. The corresponding diameters were assigned the minimal value detectable by the measurement technique. One of three patients exhibited a heterogeneous response to acetylcholine with three segments constricting and two segments remaining unchanged. There were no dilating segments in this group. The corresponding responses to nitroglycerin for these 10 segments are shown in Fig. $2 \mathrm{~B}$. In two patients, all five segments that had constricted to acetylcholine, dilated to nitroglycerin $(29 \pm 19 \%$ increase in luminal diameter $)(P<0.05)$. In the third patient, all five segments, including the segments that had constricted to acetylcholine, failed to significantly dilate to nitroglycerin. Coronary arteriograms of a patient with irregular vessels demonstrating severe paradoxical constriction to acetylcholine and preserved dilation to nitroglycerin are shown in Fig. 5.
When coronary responses to acetylcholine were analyzed by segments no apparent differences were observed in the behavior of proximal as compared to distal segments.

Clinical correlations. The clinical profiles of each patient and the angiographic appearance and functional response of the coronary arteries are presented in Table I. While larger numbers of patients may be required to reach definitive conclusions, the clinical features examined did not appear to predict the presence of vascular disease as assessed functionally with acetylcholine and anatomically by the appearance of luminal irregularities. In particular, the presence of acute myocardial rejection, current or in the past, did not appear to correlate with the development of epicardial coronary disease.

\section{Discussion}

Acetylcholine, an endothelium-dependent vasodilator acted paradoxically as a potent vasoconstrictor in many angiographically normal arteries and in all irregular arteries at 1-2 yr after transplantation. Normal physiologic dilation was seen in only a few segments of angiographically normal vessels and not in any irregular vessel segments. Nitroglycerin, which acts directly on vascular smooth muscle, dilated nearly all vessels, suggesting that the paradoxical response of transplant graft coronary arteries to acetylcholine is likely mediated by dysfunction of endothelium. This impairment appeared to be diffuse in most patients, but occasional heterogeneous segmental responses indicate that the process resulting in endothelial dysfunction can be focal. This study demonstrates that musca- 


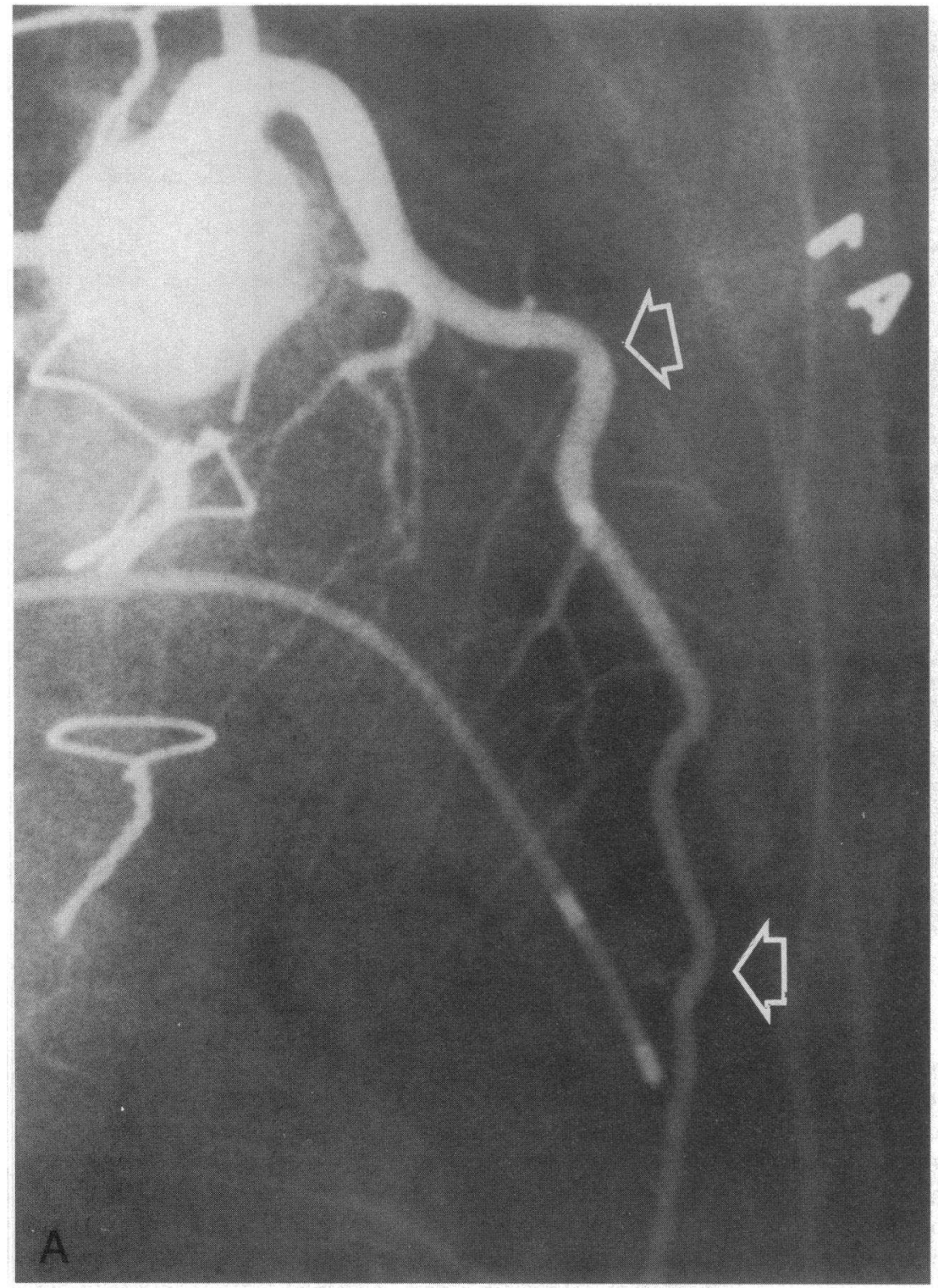

Figure 4. Coronary arteriograms in a patient with angiographically smooth coronary arteries and a constrictor response to acetylcholine. $(A)$ Left anterior descending coronary artery (arrows) under baseline condition. $(B)$ Constriction in response to $10^{-6} \mathrm{M}$ acetylcholine. $(C)$ Dilation in response to $40 \mu \mathrm{g}$ of nitroglycerin. rinic cholinergic vasodilation is impaired in some angiographically normal and nearly all "minimally diseased" coronary arteries of heart transplant patients, a population prone to accelerated graft atherosclerosis.

The development of rapidly progressive coronary artery disease in the transplanted heart is detectable by coronary arteriography in at least $40 \%$ of recipients by $2.5-5$ yr (1-3). While the disease process may be initiated by immunologic mechanisms, the histologic features of this disease bear a close resemblance to naturally occurring atherosclerosis. The changes consist of myointimal proliferation, infiltration of monocytes, the deposition of lipids in the thickened intima and deposition of collagen leading to fibrosis $(14,15)$.

The presence of even severe coronary disease may be difficult to detect in this patient population due to a lack of anginal symptoms in patients with noninnervated hearts. This prob- lem is compounded further by difficulties in interpreting thallium-201 images in patients who have undergone cardiac transplantation. Fixed image defects suggesting the presence of myocardial fibrosis may result from episodes of myocardial rejection and even reversible defects have in some cases been attributed to immunologically mediated small vessel disease $(16,17)$. Conversely, focal defects may not appear when transplant associated epicardial coronary disease is extensive and diffuse. Lastly, routine coronary arteriography may have a low sensitivity in detecting and quantifying graft atherosclerosis due to the "diffuse, tubular" nature of this process $(18,19)$.

An understanding of the role of endothelium in the control of vascular tone, its role as an antithrombogenic surface and its function in regulating vascular smooth muscle proliferation have evolved rapidly over the past decade (20). Furchgott and others have shown that a large number of arterial dilators, 


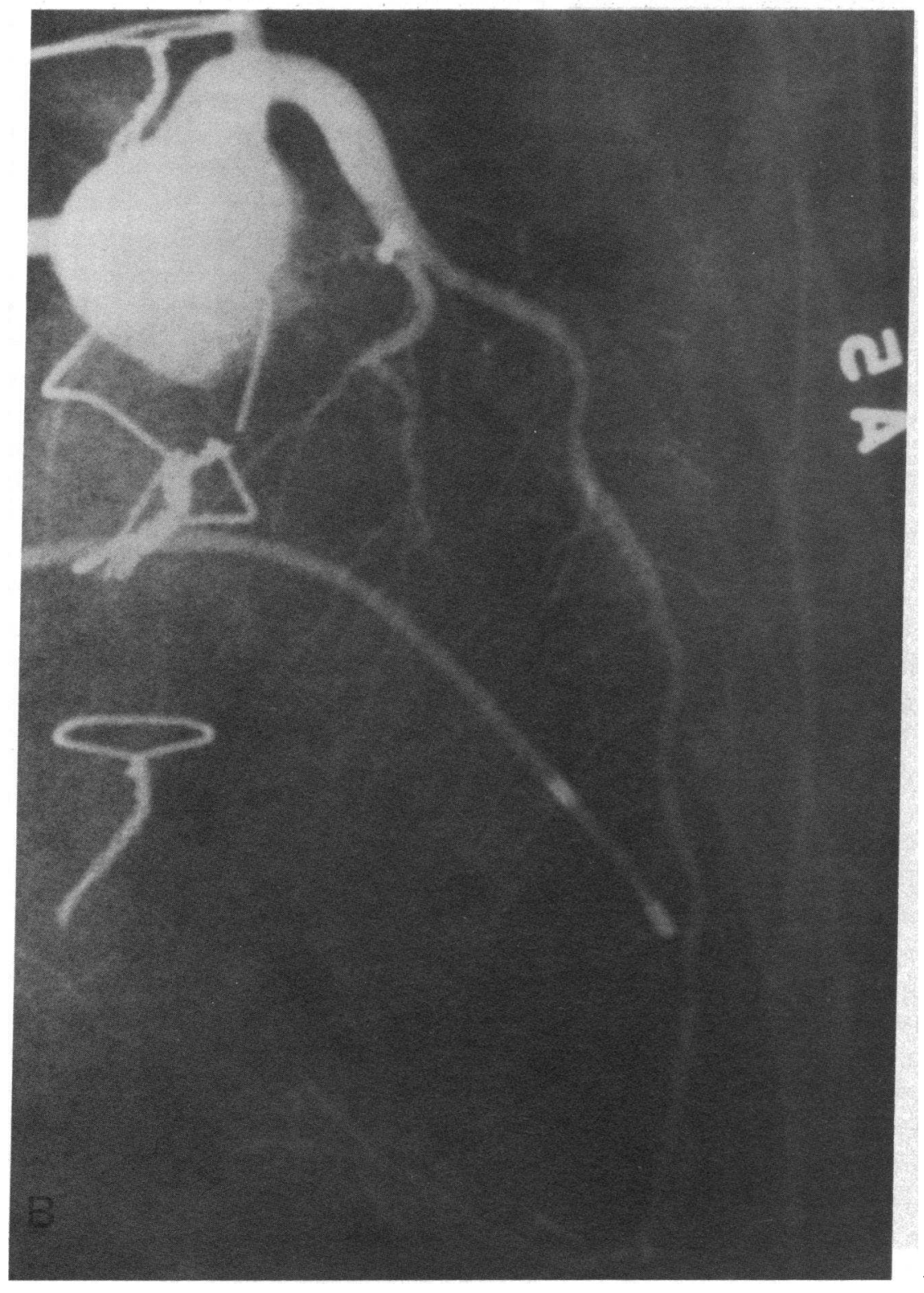

Figure 4 (Continued)

including muscarinic cholinergic agonists such as acetylcholine, relax blood vessels indirectly by stimulating the release of an endothelium-derived relating factor or factors (4-8). It has been shown in isolated vessel segments that when the endothelium is removed, the release of the endothelium-derived relaxing factor is lost, and the normal vasodilator response to acetylcholine is instead replaced by paradoxical vasoconstriction resulting from the direct effects of this agent on vascular smooth muscle. We have shown previously, that the normal response of a human coronary artery in situ in response to acetylcholine is vasodilatation (9). Vascular relaxation in response to acetylcholine has been more difficult to demonstrate in studies using human coronary artery rings in vitro, but when human coronary arteries were freshly obtained from a population free of serious atherosclerosis, relaxant effects of acetylcholine were observed (10). We (9) and others (10) have shown in patients that the endothelial dysfunction associated with the development of coronary atherosclerosis extends to a loss of vasodilator function and may result in the hypercontractility of diseased coronary arteries. In the present study, we took advantage of this functional approach to determine the frequency of endothelial dysfunction in a population of patients prone to developing coronary atherosclerosis.

This study indicates that by 1-2 yr after transplantation, endothelium-dependent relaxation is impaired in the majority of graft coronary arteries, although the relationship between the lack of appropriate vasodilation and the histopathologic features of these vessels could not be established. It is likely that in the angiographically irregular vessels the endothelial defect is due to early atherosclerosis. The findings in the angio- 


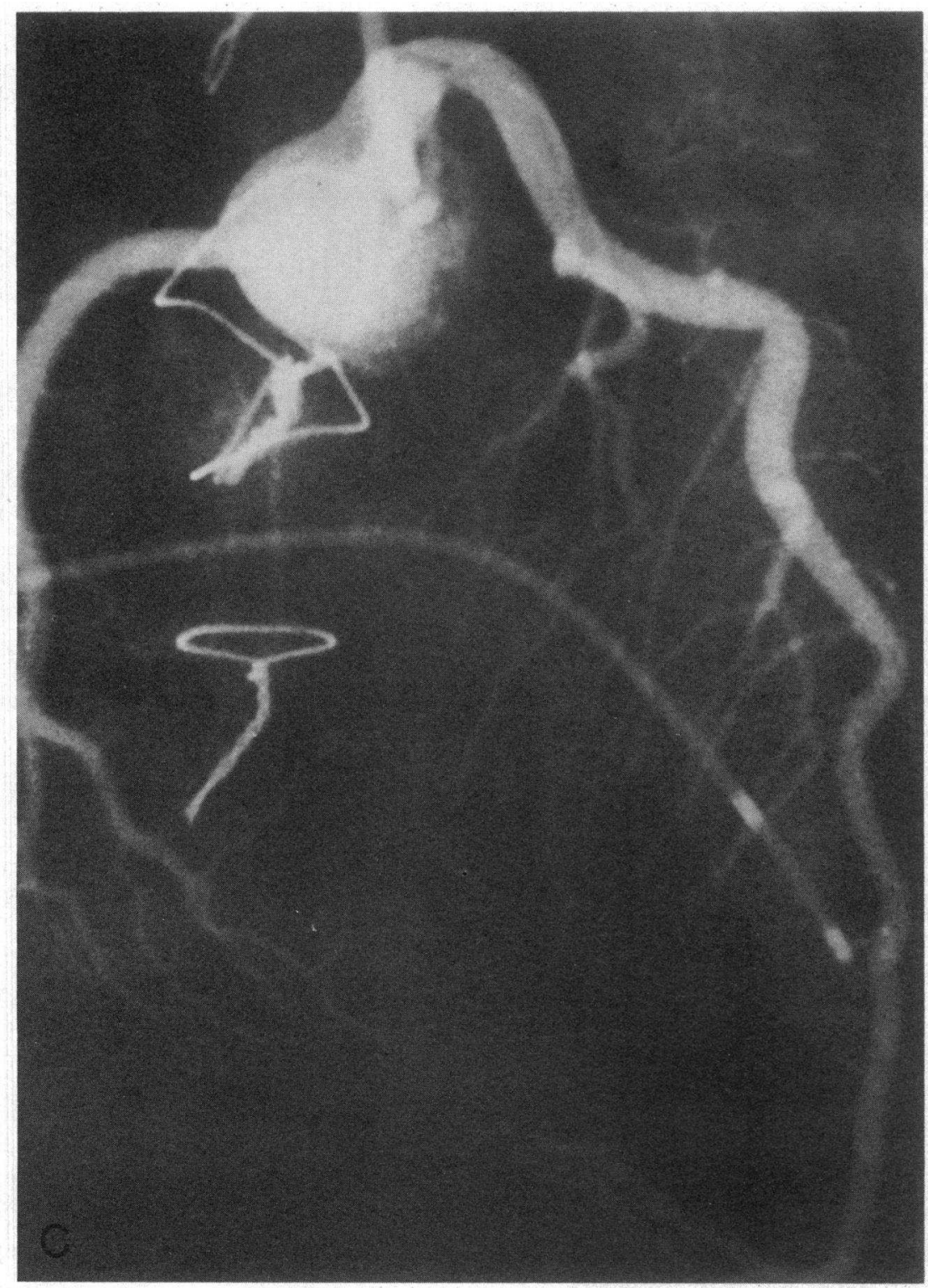

Figure 4 (Continued)

graphically smooth vessels lend credence to our previous suggestion that endothelial dysfunction in man may be manifest early in the course of coronary artery disease. The etiology of atherosclerosis in these vessels may be immune mediated. While the immune process can physically alter endothelial cell morphology (21), the capacity of endothelium to modulate its normal function has also been demonstrated in cultured cells, especially in relation to a chemical mediator of the immune process, interleukin $1(22,23)$. The inducibility by interleukin 1 of both monocyte adhesiveness (23) and the expression of procoagulant activity (22) illustrates the potential of endothelial dysfunction even in the absence of severe morphological changes. Mechanisms other than dysfunction of the endothelium that may explain the data presented in this study include increased sensitivity of vascular smooth-muscle cells to the constrictor effects of acetylcholine in diseased vessels and a defect in the coupling mechanisms between endothelium-derived relaxing factor (or factors) and smooth-muscle cells.
Additional factors that might contribute to the development of graft atherosclerosis have been sought but not found to have an important role. The disease is as frequent in the recipients whose underlying cardiac disease was cardiomyopathy as in those who had atherosclerotic disease, and its occurrence has little correlation with indexes of myocardial rejection or with the existence of the classic risk factors (1). Our study extends these previous findings to suggest that a similar lack of relationship may exist between these factors and the development of endothelial dysfunction, although a larger sample size will be necessary to ascertain this observation.

In summary, the results suggest that impaired endothelium-mediated coronary vasodilation is a common early finding in cardiac transplant patients, even in the absence of angiographic evidence of atherosclerosis. The angiographic assessment of endothelium-dependent vasodilator responses may provide for early detection and assessment of progression of coronary artery disease in heart transplant patients. Studies of 

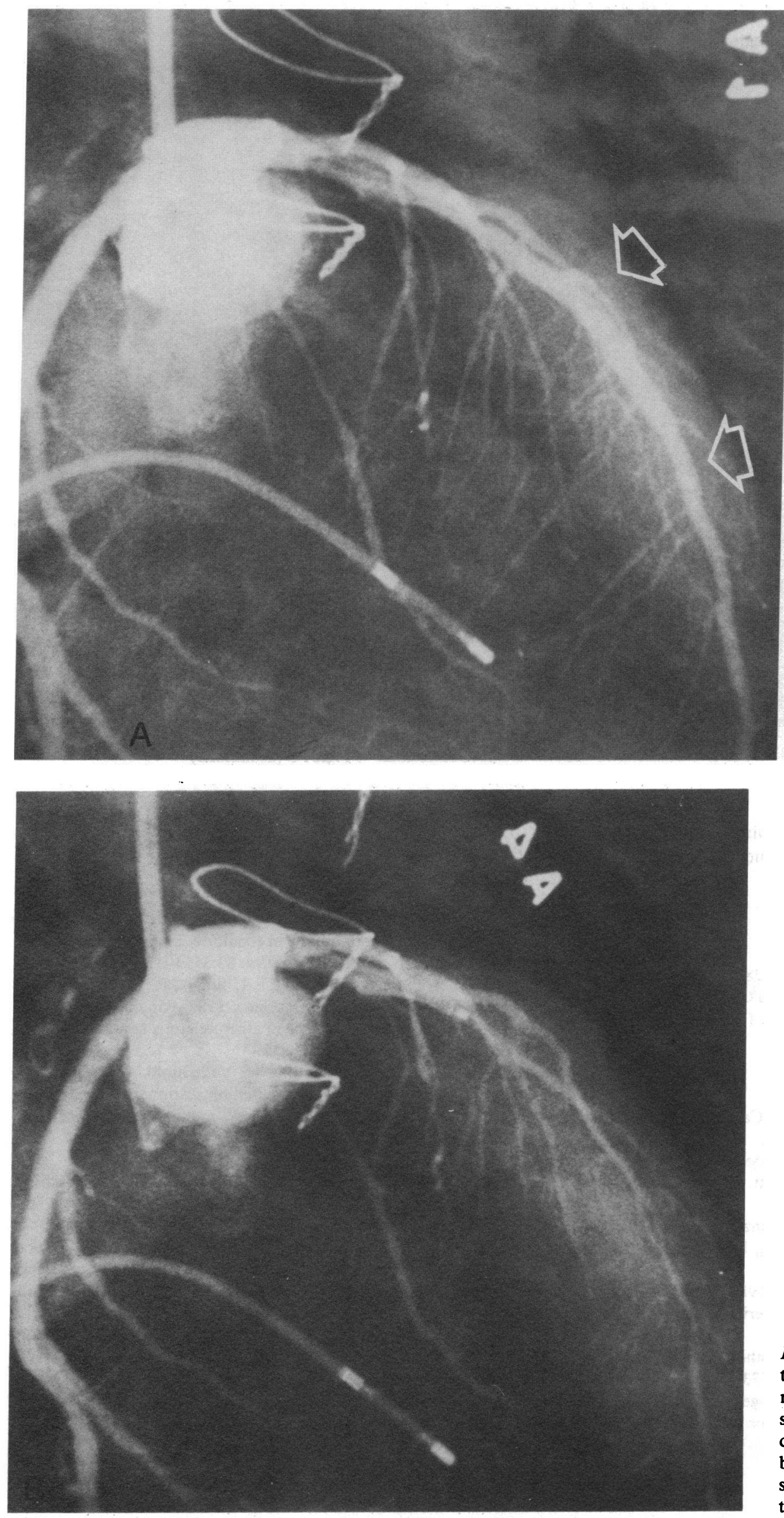

Figure 5. Coronary arteriograms in a patient with angiographically irregular coronary arteries and a marked constrictor response to acetylcholine. $(A)$ Left anterior descending coronary artery (arrows) under baseline conditions. $(B)$ Constriction in response to $10^{-7} \mathrm{M}$ acetylcholine. $(C)$ Dilation in response to $40 \mu \mathrm{g}$ of nitroglycerin. 


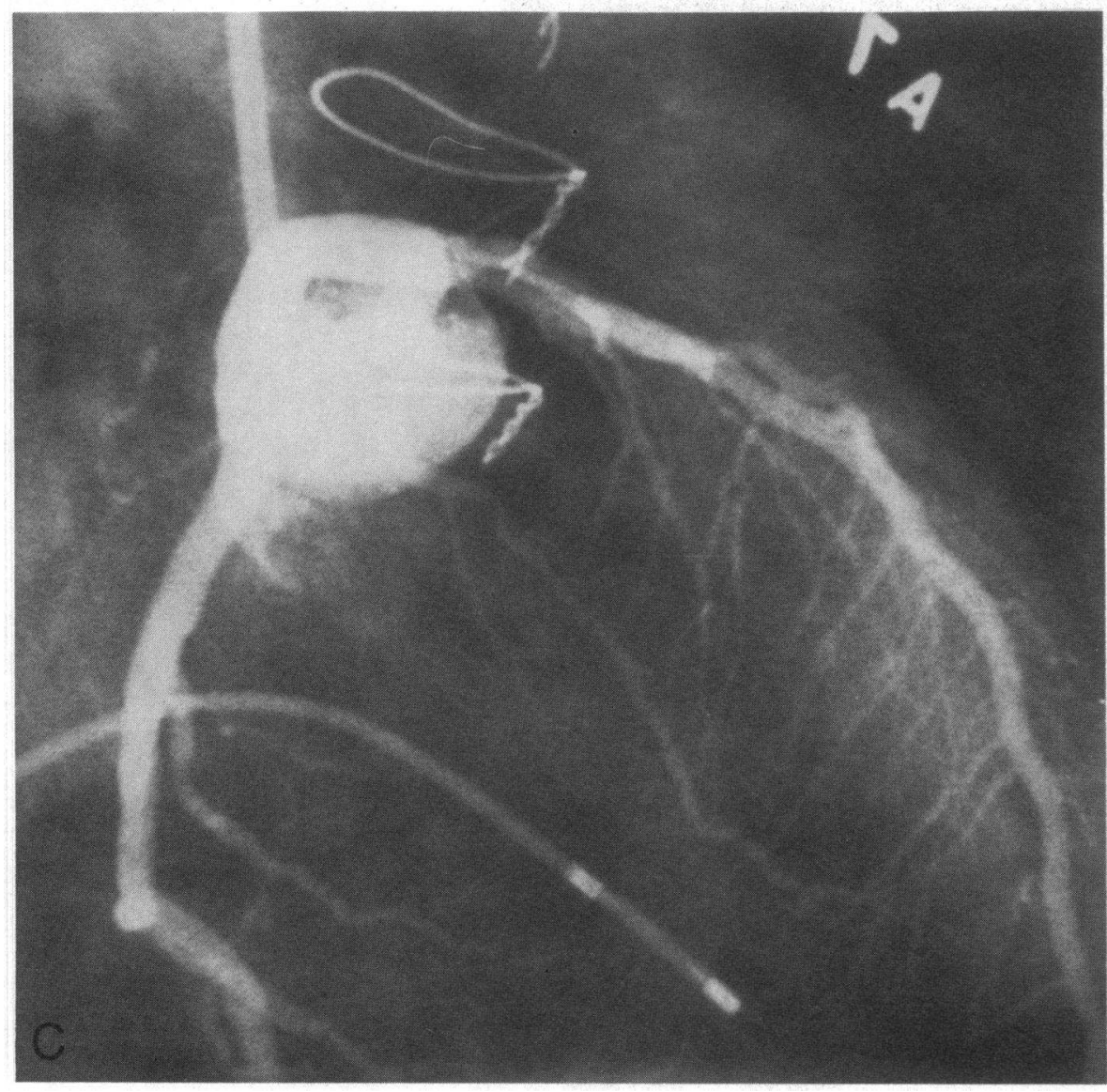

Figure 5 (Continued)

accelerated graft coronary disease may also provide a useful experimental model of interventions aimed at reversing atherosclerosis.

\section{Acknowledgments}

Supported in part by research grants HL-36028 and HL-35295 from the National Institutes of Health. Dr. Ganz is a Clinical Investigator of the National Heart, Lung, and Blood Institute (HL-01045).

\section{References}

1. Schroeder, J. S., and S. A. Hunt. 1986. Cardiac transplantation: where are we? N. Engl. J. Med. 315:961-963.

2. Nitkin, R. S., S. A. Hunt, and J. S. Schroeder. 1985. Accelerated atherosclerosis in a cardiac transplant patient. J. Am. Coll. Cardiol. 6:243-245.

3. Cooper, D. K. C., S. T. Boyd, R. P. Lanza, and C. N. Barnard. 1983. Factors influencing survival following heart transplantation. Heart Transplant. 3:86-91.

4. Furchgott, R. F., and J. V. Zawadzski. 1980. The obligatory role of endothelial cells in the relaxation of arterial smooth muscle by acetylcholine. Nature (Lond.). 288:373-376.

5. Furchgott, R. F. 1983. Role of endothelium in responses of vascular smooth muscle. Circ. Res. 53:557-573.

6. Peach, M. J., A. L. Loeb, H. A. Singer, and J. Saye. 1985. Endothelium-derived vascular relaxing factor. Hypertension. 7:194I100.
7. Vanhoutte, P. M., and T. J. Rimele. 1983. Role of endothelium in the control of vascular smooth muscle function. J. Physiol. (Paris). 78:681-686.

8. Ganz, P., P. F. Davies, J. A. Leopold, M. A. Gimbrone, Jr., and R. W. Alexander. 1986. Short- and long-term interactions of endothelium and vascular smooth muscle in coculture. Effects on cyclic GMP production. Proc. Natl. Acad. Sci. USA. 83:3553-3556.

9. Ludmer, P. L., A. P. Selwyn, T. L. Shook, R. R. Wayne, G. H. Mudge, R. W. Alexander, and P. Ganz. 1986. Paradoxical vasoconstriction induced by acetylcholine in atherosclerotic coronary arteries. N. Engl. J. Med. 315:1046-1051.

10. Bossaller, C., G. B. Habib, H. Yamamoto, C. Williams, S. Wells, and P. D. Henry. 1987. Impaired muscarinic endothelium-dependent relaxation and cyclic guanosine 5 -monophosphate formation in atherosclerotic human coronary artery and rabbit aorta. J. Clin. Invest. 79:170-174.

11. Ganz, W., K. Tamura, H. S. Marcus, R. Donoso, S. Yashida, and H. J. C. Swan. 1971. Measurements of coronary sinus blood flow by continuous thermodilution in man. Circulation. 44:181-195.

12. Spears, J. R., T. Sandor, A. V. Als, M. Malagold, J. E. Markis, W. Grossman, J. R. Serur, and S. Paulin. 1983. Computerized image analysis for quantitative measurement of vessel diameter from cineangiograms. Circulation. 68:453-461.

13. Gardiner, G. A., M. F. Meyerovitz, L. M. Boxt, D. P. Harrington, R. H. Taus, K. R. Kandarpa, P. Ganz, and A. P. Selwyn. 1986. Selective coronary angiography using a power injector. Am. J. Radiol. 146:831-833.

14. Uys, C. J., and A. G. Rose. 1982. Cardiac transplantation: Aspects of pathology. Pathol. Annu. 17:147-178. 
15. Kosek, J. C., C. Bieber, and R. R. Lower. 1971. Heart graft arteriosclerosis. Transplant. Proc. 3:512-514.

16. McKillop, J. H., M. Billingham, J. S. Schroeder, and I. R. McDougall. 1982. Correlation of an abnormal rest 201-T1 myocardial image: Pathological findings in cardiac transplant recipients. Eur. $J$. Nucl. Med. 7:243-247.

17. McKillop, J. H., and M. L. Goris. 1981. Thallium-201 myocardial imaging in patients with previous cardiac transplantation. Clin. Radiol. 32:447-449.

18. Hess, M. L., A. Mastillo, T. Mohanakumar, M. J. Cowley, G. Vetrovac, S. Szentpetery, T. C. Wolfgang, and R. R. Lower. 1983. Accelerated atherosclerosis in cardiac transplantation: Role of cytotoxic B-Cell antibodies and hyperlipidemia. Circulation. 68(Suppl. II):II-94-II-101.

19. Wohlgelernter, D., L. W. Stevenson, and R. Brunken. 1986.
Reversal of ischemic myocardial dysfunction by PTCA in a cardiac transplant patient. Am. Heart. J. 112:837-839.

20. Davies, P. F. 1986. Biology of disease. Vascular cell interactions with special reference to the pathogenesis of atherosclerosis. Lab. Invest. 55:5-24.

21. Brasile, L., T. Zerbe, B. Rabin, J. Clarke, A. Abrams, and J. Cerilli. 1985. Identification of the antibody to vascular endothelial cells in patients undergoing cardiac transplantation. Transplantation. 40:672-675.

22. Bevilacqua, M. P., J. S. Pober, G. R. Majeau, R. S. Cotran, and M. A. Gimbrone, Jr. 1984. Interleukin 1 (IL-1) induces biosynthesis and cell surface expression of procoagulant activity in human vascular endothelial cells. J. Exp. Med. 160:618-623.

23. Di Corleto, P., and C. de la Motte. 1985. Characterization of the adhesion of the human monocytic cell line U937 and cultured endothelial cells. J. Clin. Invest. 75:1153-1161. 